\title{
Using Forward-Backward Drell-Yan Asymmetry in PDF Determinations
}

\author{
Juri Fiaschi*广 \\ Institut für Theoretische Physik, Westfälische Wilhelms-Universität Münster, \\ Wilhelm-Klemm-Straße 9, D-48149 Münster, Germany \\ E-mail: fiaschi@uni-muenster.de
}

\section{Elena Accomando}

School of Physics \& Astronomy, University of Southampton, Southampton SO17 1BJ, UK

E-mail: e.accomando@soton.ac.uk

\section{Francesco Hautmann}

Theoretical Physics Department, University of Oxford, Oxford OX1 3NP, UK

CERN, Theoretical Physics Department, CH 1211, Geneva, Switzerland

E-mail: hautmannethphys.ox.ac.uk

\section{Stefano Moretti}

School of Physics \& Astronomy, University of Southampton, Southampton SO17 1BJ, UK

E-mail: s.moretti@soton.ac.uk

We study the impact of the inclusion of Neutral Current (NC) DY data from LHC mapped in the Forward-Backward Asymmetry $\left(A_{\mathrm{FB}}\right)$ observable on PDF uncertainties, using the open source platform $x F i t t e r$. We find that $A_{\mathrm{FB}}$ enables new PDF sensitivity at current and future luminosity stages of LHC.

XXVII International Workshop on Deep-Inelastic Scattering and Related Subjects - DIS2019 8-12 April, 2019

Torino, Italy

\footnotetext{
${ }^{*}$ Speaker.

${ }^{\dagger}$ On behalf of the xFitter developer's team.
} 
MS-TP-19-16

CERN-TH-2019-100

\section{Introduction}

The current and future runs at the LHC will deliver a conspicuous amount of data, which will be used to extend searches for Beyond Standard Model (BSM) physics in the high energy region and will allow precise measurements to test the Standard Model (SM) in the low energy regime. The Parton Distribution Functions (PDFs) are a necessary fundamental tool that is required in order to compare the data with theoretical predictions. The precision in the determination of the PDFs is a crucial factor in the evaluation of theoretical systematics. Improving the determination of the PDFs is therefore an important task, which will be carried out by adding new LHC data and by including additional observable that would serve the purpose.

In this work, we study the impact from the inclusion of Drell-Yan (DY) di-lepton data in the form of Forward-Backward Asymmetry $\left(A_{\mathrm{FB}}\right)$ in the determination of PDFs. It was already shown that this observable can provide constraints on the PDFs [1,2] and here we quantify this effect by using the open source platform xFitter [3] for the profiling of various sets of PDFs [4]. We employ $A_{\mathrm{FB}}$ pseudodata with a statistical precision corresponding to different integrated luminosities ranging from $30 \mathrm{fb}^{-1}$ to $3000 \mathrm{fb}^{-1}$.

\section{Details of the analysis}

We started by implementing a suitable $\mathrm{C}++$ code for the calculation of the $A_{\mathrm{FB}}$ at Leading Order (LO) and integrating it into the xFitter environment. Drell-Yan production of di-lepton pair is mediated by the exchange of a photon or a $Z$ boson, and the $A_{\mathrm{FB}}$ is defined as

$$
A_{\mathrm{FB}}^{*}=\frac{d \sigma / d M\left(\ell^{+} \ell^{-}\right)\left[\cos \theta^{*}>0\right]-d \sigma / d M\left(\ell^{+} \ell^{-}\right)\left[\cos \theta^{*}<0\right]}{d \sigma / d M\left(\ell^{+} \ell^{-}\right)\left[\cos \theta^{*}>0\right]+d \sigma / d M\left(\ell^{+} \ell^{-}\right)\left[\cos \theta^{*}<0\right]} .
$$

At LO, the angle $\theta^{*}$ is defined according to the direction of the incoming quark, which is assumed to be given by the direction of the boost of the final state di-lepton pair $[5,6,7,8,9]$.

Subsequently we included NLO corrections to the observable, employing suitable NLO grids obtained with MadGraph5_aMC@NLO [10] interfaced to APPLgrid [11] through aMC fast [12]. At NLO the angle $\theta^{*}$ is defined is defined in the Collins-Soper (CS) frame [13] as

$$
\cos \theta^{*}=\frac{p_{Z, \ell \ell}}{M_{\ell \ell}\left|p_{Z, \ell \ell}\right|} \frac{p_{1}^{+} p_{2}^{-}-p_{1}^{-} p_{2}^{+}}{\sqrt{M_{\ell \ell}^{2}+p_{T, \ell \ell}^{2}}},
$$

with $p_{i}^{ \pm}=E_{i} \pm p_{Z, i}$, the index $i=1,2$ labeling the positive and negative charged lepton respectively. $E$ and $p_{Z}$ are the energy and the $z$-components of the leptonic four-momentum, respectively; $p_{Z, \ell \ell}$ is the di-lepton $z$-component of the momentum and $p_{T, \ell \ell}$ is the di-lepton transverse momentum.

The cross sections appearing in Eq. 2.1 are calculated in the fiducial region, i.e. applying the standard kinematical cuts to simulate the detector acceptance $\left(\left|\eta_{\ell}\right|<2.5\right.$ and $\left.p_{T}^{\ell}>20 \mathrm{GeV}\right)$. A set of datafiles has been generated for each PDF set under analysis. They contain the predictions for 
the observable in the invariant mass range $45 \mathrm{GeV}<M_{\ell \ell}<200 \mathrm{GeV}$ with a bin width of $2.5 \mathrm{GeV}$, and the projected statistical error, obtained following

$$
\Delta A_{\mathrm{FB}}^{*}=\sqrt{\frac{1-A_{\mathrm{FB}}^{*}}{N}},
$$

where the number of events $N$ includes an acceptance times efficiency factor of the order of $\sim 20 \%$ [14] and NNLO QCD corrections included by adopting a mass dependent $k$-factor $[15,16]$. The datafiles have been generated fixing the collider centre-of-mass energy at $13 \mathrm{TeV}$ and for three values of integrated luminosity $\left(30 \mathrm{fb}^{-1}, 300 \mathrm{fb}^{-1}\right.$ and $\left.3000 \mathrm{fb}^{-1}\right)$. Furthermore we also considered the effects on the PDF profiling from the application of rapidity cuts on the di-lepton system. For this purpose, datafiles have been generated with $\left|y_{\ell \ell}\right|>0$ (no rapidity cut), $\left|y_{\ell \ell}\right|>1.5$ and $\left|y_{\ell \ell}\right|>4$.0. The profiling technique [17] is based on minimizing $\chi^{2}$ between data and theoretical predictions. It assumes that the new data are compatible with the theoretical predictions using the existing PDF set and, under this assumption, the central values of the data points are set to the central values of the theoretical predictions. No theoretical uncertainties except the PDF uncertainties are considered when calculating the $\chi^{2}$.

\section{Profiled PDFs with $A_{\mathrm{FB}}$}

In this section we present the results for the profiling of various PDF sets when including $A_{\mathrm{FB}}$ measurements. In Fig. 1 we show the results of the profiling on the CT14nnlo [18] PDF set. The error bands obtained with this PDF set have been rescaled to 68\% CL for a consistent comparison with the results obtained for other PDF sets. We display the error bands for the normalised distribution of $u$-valence, $d$-valence, $u$-sea and $d$-sea quarks. The profiled curves are obtained including $A_{\mathrm{FB}}$ data with a statistical error corresponding to integrated luminosities of $30 \mathrm{fb}^{-1}$ (blue), 300 $\mathrm{fb}^{-1}$ (green) and $3000 \mathrm{fb}^{-1}$ (orange). The $u$-valence and the $d$-valence quark distributions show the largest sensitivity to the new data, especially in the region of intermediate and low momentum fraction $x$. Sea quark distributions also exhibit some improvement, particularly in the region of intermediate $x$ for high luminosity.
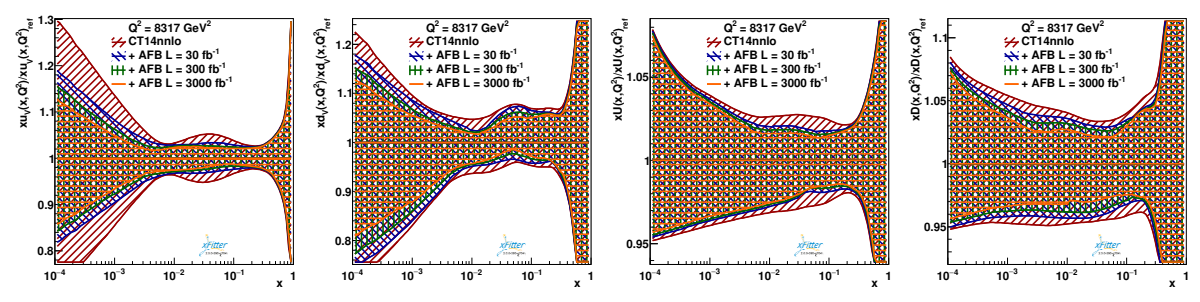

Figure 1: Original and profiled distributions for the normalised ratios of (left to right) $u$-valence, $d$-valence, $u$-sea and $d$-sea quarks of the CT14nnlo PDF set, obtained with $A_{\mathrm{FB}}^{*}$ pseudodata at different luminosities.

In a similar way, in Fig. 2 we show the profiled curves for the PDF sets (rows from top to bottom) NNPDF3.1nnlo (Hessian set) [19], MMHT2014nnlo [20], ABMP16nnlo [21] and HERA2.0nnlo (EIG) [22], obtained including $A_{\mathrm{FB}}$ data with a projected statistical error corresponding to an integrated luminosity of $300 \mathrm{fb}^{-1}$. The ABMP16nnlo and HERA2.0nnlo sets appear to be the most sensitive to the new data, especially in the distributions of $u$-valence and $d$-valence quarks in the region 
of low and intermediate $x$, while less improvement is visible in the sea quarks distributions. The NNPDF3.1nnlo and MMHT2014nnlo sets also show some improvement in the in the region of low to intermediate $x$ for the valence quarks distribution, while also a visible improvement is observed in the $u$-sea (especially for NNPDF3.1nnlo) and in the $d$-sea (especially for MMHT2014nnlo) error bands.
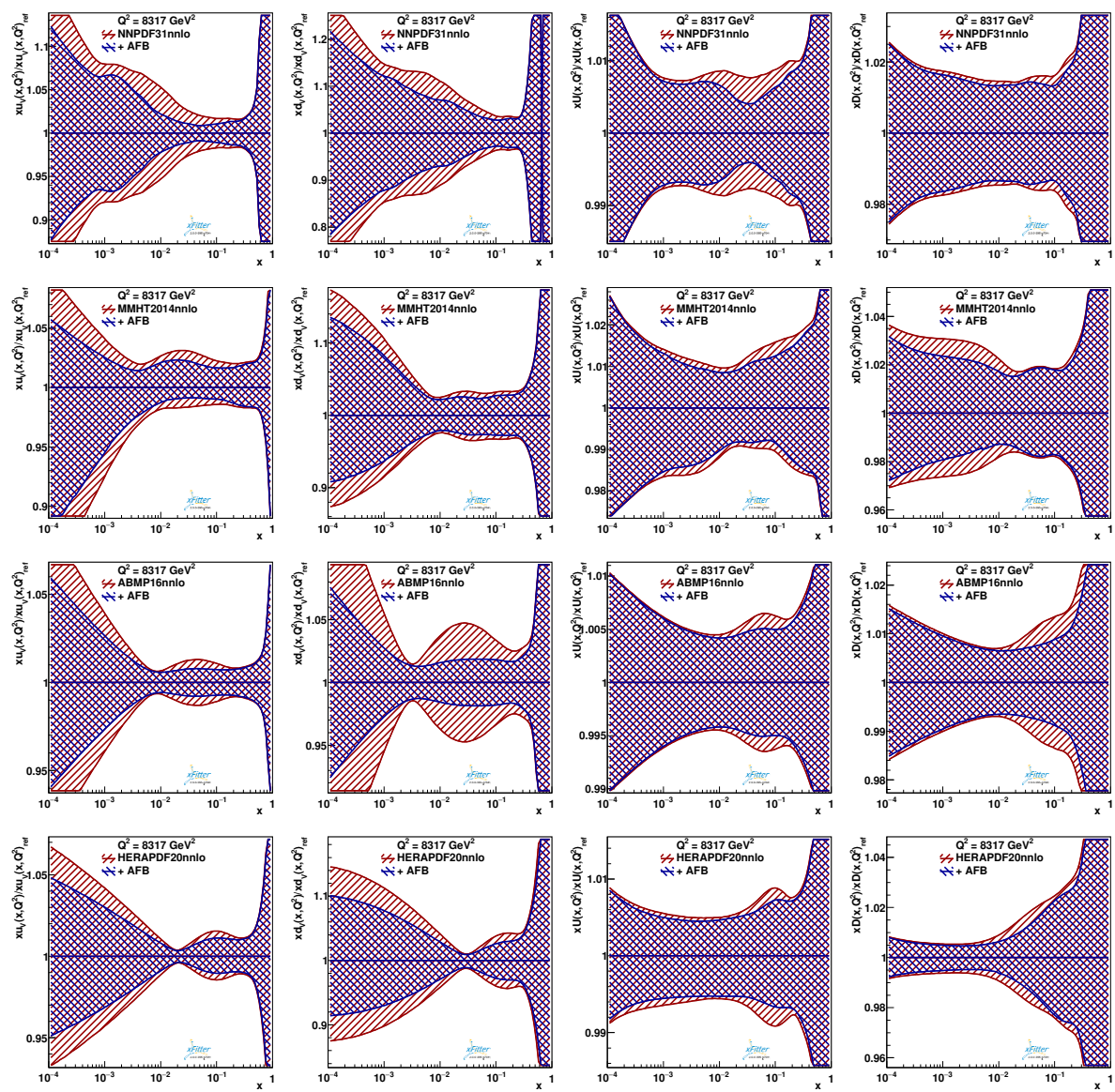

Figure 2: Original and profiled distributions for the normalised ratios of (left to right) $u$-valence, $d$-valence, $u$-sea and $d$-sea quarks, obtained with $A_{\mathrm{FB}}^{*}$ pseudodata at $\mathrm{L}=300 \mathrm{fb}^{-1}$. Distributions are shown for the PDF sets (top to bottom) NNPDF3.1nnlo, MMHT2014nnlo, ABMP16nnlo and HERA2.0nnlo.

In Fig. 3 we show the effects of the application of low rapidity cuts on the data on the profiled error bands of the HERA2.0nnlo PDF set, using $A_{\mathrm{FB}}$ data with a statistical error corresponding to an integrated luminosity of $3000 \mathrm{fb}^{-1}$. We display the results for the distribution of $u$-valence, $d$-valence and their combination $(u+d)$-valence quarks, using data where rapidity cuts of $\left|y_{\ell \ell}\right|>0$ (blue), $\left|y_{\ell \ell}\right|>1.5$ (green) and $\left|y_{\ell \ell}\right|>4.0$ (orange) have been applied. In the latter case the analysis is performed using $A_{\mathrm{FB}}^{*}$ predictions at $\mathrm{LO}$ and pseudodata generated in the invariant mass range $80 \mathrm{GeV}<M_{\ell \ell}<200 \mathrm{GeV}$ and with a bin size of $1 \mathrm{GeV}$. Furthermore, in order to explore the very high rapidity region, we have extended the detector acceptance region up to pseudorapidity $\left|\eta_{\ell}\right|<5$. When comparing the profiled error bands obtained with $\left|y_{\ell \ell}\right|>0$ and $\left|y_{\ell \ell}\right|>1.5$, we observe a remarkable improvement in the distribution of the valence quarks, especially in the region of low $x$, which is then reflected in the combination $(u+d)$-valence. On the other hand, the curve obtained 

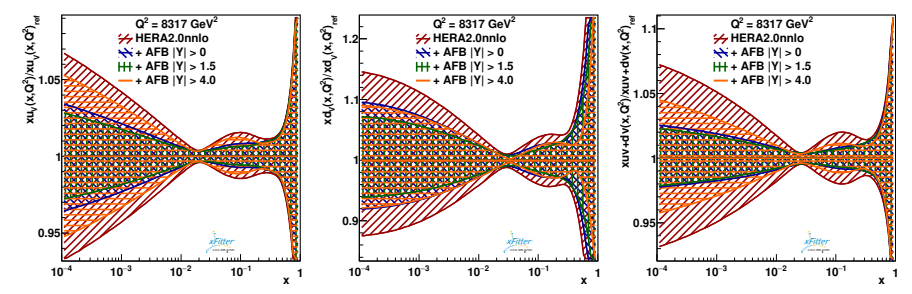

Figure 3: Original (red) and profiled distributions for the normalised ratios of $u$-valence, $d$-valence and $(u+d)$-valence, obtained with $A_{\mathrm{FB}}^{*}$ pseudodata at $\mathrm{L}=3000 \mathrm{fb}^{-1}$ applying rapidity cuts of $\left|y_{\ell \ell}\right|>0$ (blue), $\left|y_{\ell \ell}\right|>1.5$ (green) and $\left|y_{\ell \ell}\right|>4$ (orange) respectively. In the latter case the analysis is performed at LO and acceptance region of the detector has been enlarged up to $\left|\eta_{\ell}\right|<5$.

with a rapidity cut $\left|y_{\ell \ell}\right|>4.0$ shows an overall weaker profiling compared to the previous cases. This is mostly due to the reduced statistic consequence of the stronger phase space cut. However here the data allows to access higher $x$ and we can appreciate a reduction of the uncertainty error bands concentrated in that region.

\section{Conclusions}

The new data from the LHC Runs 2 and the upcoming Run 3 and HL-LHC stages will provide important information to constrain non-perturbative QCD effects from PDFs. DY data in particular can be exploited both in the form of cross section and asymmetry. The latter contains a complementary information with respect to the cross section and it also encodes information on the lepton polar angle, or pseudorapidity. We have considered the constraints on PDFs from the inclusion of $A_{\mathrm{FB}}$ data, implementing this observable in the XFitter framework and obtaining profiled curves to assess the impact of the new data. Suitable pseudodata have been generated for such purpose for various PDF sets, projecting current and future statistical precision of the measurements with integrated luminosities of $30 \mathrm{fb}^{-1}, 300 \mathrm{fb}^{-1}$ and $3000 \mathrm{fb}^{-1}$. We have observed a significant improvement of PDF uncertainties, especially in the valence quarks distribution in the low and intermediate $x$ region. Some amelioration has been also obtained in the sea quarks distributions when employing high statistics data. We have evidenced substantial differences in the results obtained for the different PDF sets. Furthermore, we have studied the impact of rapidity cuts on the di-lepton system, and we noted that by these means we can further improve PDF distributions in the high $x$ region.

\section{References}

[1] E. Accomando, J. Fiaschi, F. Hautmann and S. Moretti, Neutral current forward backward asymmetry: from $\theta_{W}$ to PDF determinations, Eur. Phys. J. C78 (2018) 663 [1805. 09239].

[2] E. Accomando, J. Fiaschi, F. Hautmann and S. Moretti, Constraining Parton Distribution Functions from Neutral Current Drell-Yan Measurements, Phys. Rev. D98 (2018) 013003 [1712 . 06318].

[3] S. Alekhin et al., HERAFitter, Eur. Phys. J. C75 (2015) 304 [1410 . 4412].

[4] E. Accomando, J. Fiaschi, F. Hautmann, S. Moretti, the xFitter Developers' team: H. Abdolmaleki, V. Bertone et al., PDF Profiling Using the Forward-Backward Asymmetry in Neutral Current Drell-Yan Production [1907.07727]. 
[5] M. Dittmar, Neutral current interference in the TeV region: The Experimental sensitivity at the LHC, Phys. Rev. D55 (1997) 161 [hep-ex/9606002].

[6] T. Rizzo, Indirect Searches for Z'-like Resonances at the LHC, JHEP 08 (2009) 082 [090 4 . 2534].

[7] E. Accomando, A. Belyaev, J. Fiaschi, K. Mimasu, S. Moretti and C. Shepherd-Themistocleous, Forward-backward asymmetry as a discovery tool for Z' bosons at the LHC, JHEP 01 (2016) 127 [1503.02672].

[8] E. Accomando, J. Fiaschi, F. Hautmann, S. Moretti and C. H. Shepherd-Themistocleous, Photon-initiated production of a dilepton final state at the LHC: Cross section versus forward-backward asymmetry studies, Phys. Rev. D95 (2017) 035014 [1606.06646].

[9] E. Accomando, J. Fiaschi, F. Hautmann, S. Moretti and C. H. Shepherd-Themistocleous, The effect of real and virtual photons in the di-lepton channel at the LHC, Phys. Lett. B770 (2017) 1 [1612.08168].

[10] J. Alwall, R. Frederix, S. Frixione, V. Hirschi, F. Maltoni, O. Mattelaer et al., The automated computation of tree-level and next-to-leading order differential cross sections, and their matching to parton shower simulations, JHEP 07 (2014) 079 [1405.0301].

[11] T. Carli, D. Clements, A. Cooper-Sarkar, C. Gwenlan, G. P. Salam, F. Siegert et al., A posteriori inclusion of parton density functions in NLO QCD final-state calculations at hadron colliders: The APPLGRID Project, Eur. Phys. J. C66 (2010) 503 [0911.2985].

[12] V. Bertone, R. Frederix, S. Frixione, J. Rojo and M. Sutton, aMCfast: automation of fast NLO computations for PDF fits, JHEP 08 (2014) 166 [1406. 7693 ].

[13] J. C. Collins and D. E. Soper, Angular Distribution of Dileptons in High-Energy Hadron Collisions, Phys. Rev. D16 (1977) 2219.

[14] CMS collaboration, Search for physics beyond the standard model in dilepton mass spectra in proton-proton collisions at $\sqrt{s}=8 \mathrm{TeV}$, JHEP 04 (2015) 025 [1412.6302].

[15] R. Hamberg, W. L. van Neerven and T. Matsuura, A complete calculation of the order $\alpha_{s}^{2}$ correction to the Drell-Yan K factor, Nucl. Phys. B359 (1991) 343.

[16] R. V. Harlander and W. B. Kilgore, Next-to-next-to-leading order Higgs production at hadron colliders, Phys. Rev. Lett. 88 (2002) 201801 [hep-ph/ 0201206 ].

[17] H. Paukkunen and P. Zurita, PDF reweighting in the Hessian matrix approach, JHEP 12 (2014) 100 [1402.6623].

[18] S. Dulat, T.-J. Hou, J. Gao, M. Guzzi, J. Huston, P. Nadolsky et al., New parton distribution functions from a global analysis of quantum chromodynamics, Phys. Rev. D93 (2016) 033006 [1506. 07443 ].

[19] NNPDF collaboration, Parton distributions from high-precision collider data, Eur. Phys. J. C77 (2017) 663 [1706.00428].

[20] L. A. Harland-Lang, A. D. Martin, P. Motylinski and R. S. Thorne, Parton distributions in the LHC era: MMHT 2014 PDFs, Eur. Phys. J. C75 (2015) 204 [1412 . 3989 ].

[21] S. Alekhin, J. BlÃijmlein, S. Moch and R. Placakyte, Parton distribution functions, $\alpha_{s}$, and heavy-quark masses for LHC Run II, Phys. Rev. D96 (2017) 014011 [1701. 0 5838].

[22] ZEUS, H1 collaboration, Combination of measurements of inclusive deep inelastic $e^{ \pm} p$ scattering cross sections and QCD analysis of HERA data, Eur. Phys. J. C75 (2015) 580 [1506. 06042 ]. 